\title{
effet des vibrations transmises par le sol
}

\author{
par \\ R. Bonaz \\ SIMECSOL, Paris
}

RESUME - Les vibrations transmises par le sol agissent aussi bien sur les personnes que sur les bâtiments et les terrains. Des exemples concrets sont donnés se rapportant aux tirs d'explosifs en site sensible, à la perforation de maçonnerie ou à la circulation de véhicules civils et militaires agissant sur des monuments historiques, etc... Diverses méthodes de prévision de niveau vibratoire sont exposées et des indications pratiques sont données quant à la manière d'agir.

\section{INTRODUCTION}

Respecter l'environnement est un souci croissant pour l'aménageur ou l'entrepreneur qui se posent de plus en plus fréquemment le problème de l'évaluation des risques de nuisances et les remèdes à trouver. Ceci est particulièrement vrai pour les vibrations transmises par le sol qui peuvent mettre en cause aussi bien le confort des personnes que la sécurité des ouvrages.

Les aspects théoriques des phénomènes vibratoires dans les sols et dans les structures ont été largement traités dans la littérature ces dernières années. Il est intéressant d'apporter ici des exemples concrets, faisant état d'une expérience acquise sur le terrain à travers des cas variés.

\section{I - SOURCES DE VIBRATIONS}

Les sources de vibrations qu'on peut rencontrer dans notre environnement sont nombreuses et diverses, mais elles se classent en deux groupes distincts :

- Les excitations impulsionnelles : ce sont les phénomènes de choc créant un train d'ondes de courte durée, isolément ou en série. Les origines possibles sont le battage de pieux, les tirs d'explosif, le compactage dynamique, certaines machines - outils, etc...

- Les vibrations entretenues produisant un train d'ondes continu dans le temps dont l'origine peut être : la circulation routière ou ferroviaire, le vibrofonçage, le vibrocompactage, la perforation de maçonnerie, etc...

\section{II - EFFETS POSSIBLES}

A partir de leur source, les ondes élastiques rayonnent dans le terrain, pénètrent dans les fondations des ouvrages, puis se propagent au travers de la structure, et atteignent les occupants.

Pour évaluer les effets possibles, il y a lieu d'examiner toutes les étapes du trajet parcouru :

- dans le sol : les vibrations peuvent créer des tassements, ou même la liquéfaction, des ruptures de pentes, introduire de la fatigue (HAIMSON - 1978), ouvrir des fissures préexistantes, etc...

- dans les constructions, les conséquences sont de gravité très variable : fissuration superficielle, déplacement ou chute d'éléments de stabilité précaire (crépi, corniches, objets suspendus, etc...) mise en résonnance localisée ou générale de l'ossature, fissuration des éléments portants.

- pour les personnes, les vibrations peuvent constituer une gène pour le confort, ou même créer des perturbations physiologiques ; dans presque tous les cas, c'est la cause d'inquiétudes plus ou moins bien contrôlées ou justifiées.

\section{III - EXEMPLES}

III.l. "Monte Carlo Sun"

L'ensemble immabilier "Monte Carlo Sun" situé à MONACO à proximité de la mer, est installé sur une plateforme taillée dans un éboulis de 
pente dont la granulométrie s'étend de façon continue des argiles aux blocs de plusieurs mètres cubes. Le bâtiment principal est fondé sur 21 puits, de diamètre variant entre $2,50 \mathrm{~m}$ et $4,00 \mathrm{~m}$, et atteignant le substratum, situé à une quinzaine de mètres. L'emprise du chantier est traversée par une galerie de captage d'eau potable alimentant la ville de MONACO ; cette galerie est de section ovolide (hauteur : $2 \mathrm{~m}$, largeur : $1,30 \mathrm{~m}$ environ), et revêtue intérieurement de briques maçonnées ; elle borde l'emprise des fondations sur deux côtés et son toit est à $7,50 \mathrm{~m}$ de profondeur. Une dizaine de puits sont situés à des distances comprises entre 1 et $5 \mathrm{~m}$ de l'extrados de la galerie.

Les explosifs ont dâ être utilisés au cours de l'excavation, pour le passage des gros blocs et dans le substratum. Les ébranlements crés pouvaient mettre en cause la stabilité de la galerie de captage, dont la préservation devrait être assurée avec le maximum de sécurité. Une série de mesures effectuées au cours d'une campagne d'essais a permis de fixer des règles de tirs. L'instrumentation a été disposée dans une même section située au plus près du point de tir et comprenant 3 capteurs triaxiaux scellés au parement et répartis le long de la face la plus exposée.

Il n'a pas été possible de dégager une loi d'amortissement dans les éboulis; en effet, les blocs étant disposés de façon irrégulière, il existe probablement des cheminements privilégiés pour les vibrations, constitués par la juxtaposition de blocs dont on ne connaît à priori ni l'existence, ni la position. Ceci a conduit à réaliser au total une vingtaine de tirs d'essai.

Le fractionnement des charges, et l'utilisation de microretards a été la méthode utilisée pour réduire le niveau de vibrations et une illustration en est donnée par les oscillogrammes des tirs $n^{\circ} 10, n^{\circ} 13$ et $n^{\circ} 17$.
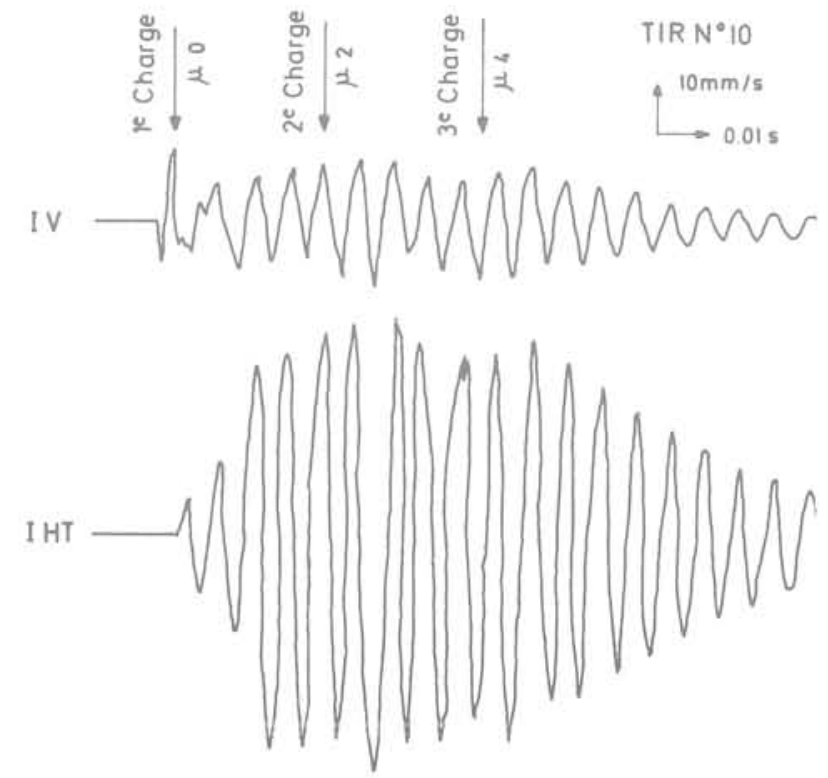

Figure 1 - Oscillogramme du tir $n^{0} 10$
- Tir no 10 : la charge totale était de $600 \mathrm{~g}$, et constitué de 3 charges instantanées de $200 \mathrm{~g}$, mise à feu avec les microretards 0,2 et 4 . L'oscillogramme montre que les ébranlements correspondant n'ont pas le temps de s'amortir entre deux mises à feu successives et qu'ils ont tendance même à s'ajouter (Figure 1).

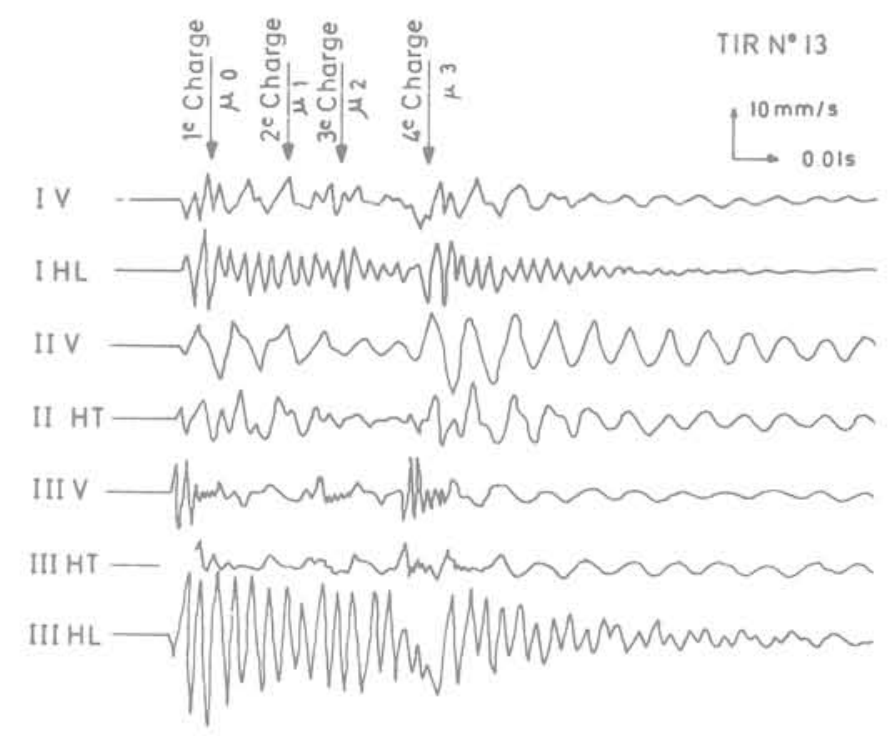

Figure 2 - Oscillogramme du tir $n^{0} 13$

- Tir $n^{0} 13$ : la charge totale était de $400 \mathrm{~g}$, et la charge instantanée a été réduite à $100 \mathrm{~g}$; l'effet des microretards commence à se faire sentir mais le délai entre chaque mise à feu est encore trop court pour permettre la pleine efficacité de la méthode (Figure 2).

- Tir $n^{0} 17$ : la charge totale de 300 g a été répartie en 6 charges de 50 g séparée par un à deux numéros de microretard. L'effet de chaque charge est clairement isolé et s'amortit suffisamment pour ne pas se cumuler avec la charge suivante (Figure 3 ).

Compte tenu du grand nombre de paramètres à prendre en compte (charge totale, charges instantanées, microretards, position relative charge / section de mesure, tir de surface, en fond de puits, dans un bloc, dans le substratum, etc...), nous ne donnerons que quelques valeurs indicatives (Tableau I).

\begin{tabular}{|c|c|c|}
\hline Charge $(\mathrm{g})$ & Distance $(\mathrm{m})$ & Vitesse $(\mathrm{mm} / \mathrm{s})$ \\
\hline $600=3 \times 200$ & 25 & 27 \\
$400=4 \times 100$ & 16,5 & 20 \\
$300=4 \times 75$ & 9,5 & 19 \\
$300=6 \times 50$ & 9,5 & 20 \\
$300=6 \times 50$ & 2,5 & 23 \\
\hline
\end{tabular}

Tableau I 


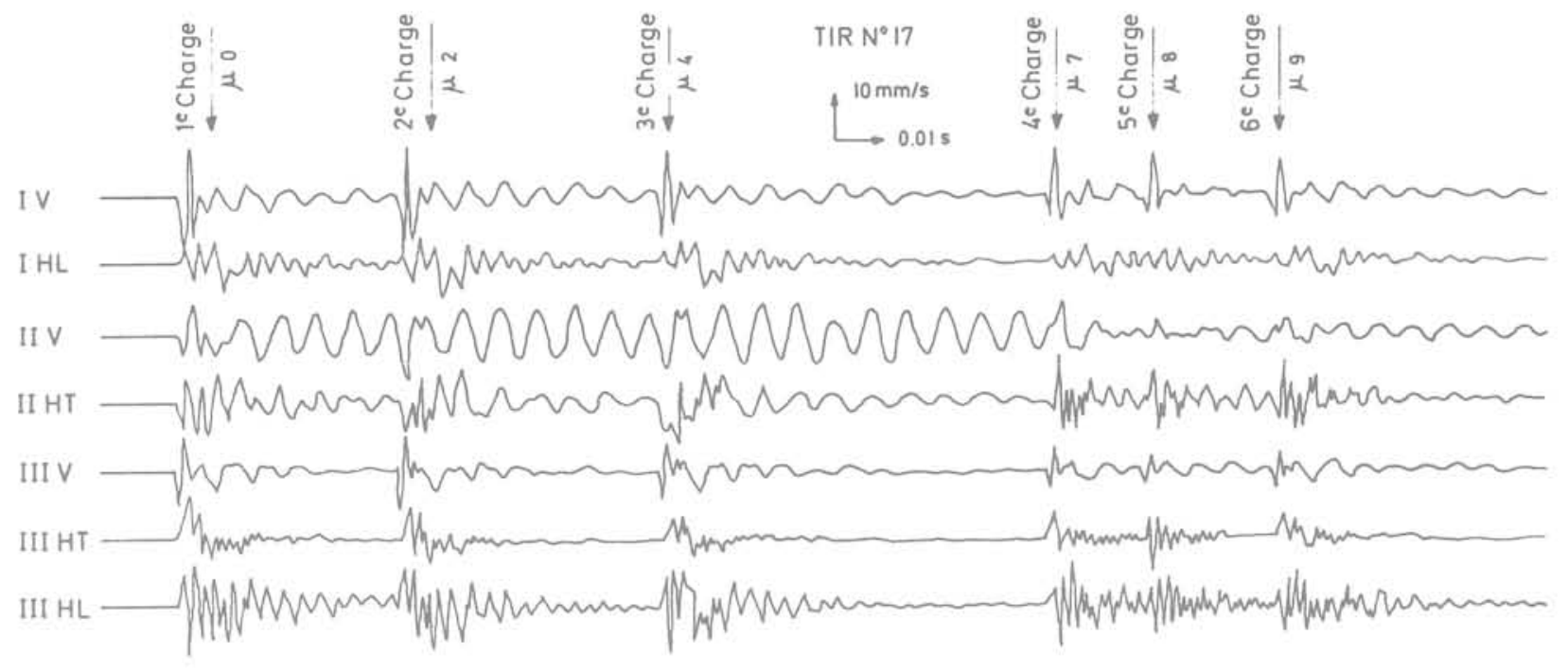

Figure 3 - Oscillogramme du tir $n^{0} 17$

Les tirs d'essai à $2,50 \mathrm{~m}$ de la galerie ont fait l'objet de précautions particulières : perforation d'une série de trous à la périphérie espacés de $0,25 \mathrm{~m}$, complétée par deux trous de décompression à l'intérieur ; de surcroit, le fond du puits étant dans la nappe, les trous périphériques ont été équipés de tubes PVC bouchés à leur base créant ainsi un vide d'air gènant la transmission des vibrations. Ces dispositions ont permis d'abaisser les amplitudes vibratoires à un niveau comparable à celui obtenu pour une distance de $9,50 \mathrm{~m}$ et plus.

Compte tenu des résultats fournis par la campagne d'essais, le seuil retenu pour la définition du plan de tir a été de $25 \mathrm{~mm} / \mathrm{s}$. Cette valeur prudente a permis cependant d'autoriser le terrassement à l'explosif tout en respectant les contraintes d'environnement.

La galerie n'a subi aucun dommage, même mineur, ni pendant les tirs d'essais ni pendant la phase des travaux.

\section{III.2. Musée de 1'Orangerie (PARIS)}

Le Musée de 1'0rangerie à PARIS, abrite les "Nymphéas", toiles de Manet qui sont directement collées sur des cloisons en briques. A la suite de mouvements de fondations, des fissures multiples sont apparues, dont certaines menaçant directement les toiles.

Au cours des travaux de confortement, les fondations ont été perforées pour la mise en place de micro-pieux; du fait de la sensibilité de l'ouvrage, un contrôle des niveaux vibratoires s'est avéré nécessaire. Les mesures ont été effectuées à l'intérieur du bâtiment, au rez de chaussée, en général dans la zone la plus proche de la source de vibrations. Les résultats sont les suivants (Tableau II) :

\begin{tabular}{|l|c|c|}
\hline \multicolumn{1}{|c|}{ Source } & $\begin{array}{c}\text { Distance } \\
(\mathrm{m})\end{array}$ & $\begin{array}{c}\text { Vitesse } \\
\text { résultante } \\
\text { (mm/s) }\end{array}$ \\
\hline $\begin{array}{l}\text { Forage à l'extérieur } \\
\text { du bâtiment : } \\
\text { - perforation de la } \\
\text { dalle de surface } \\
\text { perforation du sol } \\
\text { (meuble) }\end{array}$ & 3 & 2,5 \\
$\begin{array}{l}\text { Coups de marteau pour } \\
\text { calage de la perfora- } \\
\text { trice au sous-sol } \\
\begin{array}{l}\text { Perforation du terrain } \\
\text { (machine au sous-sol) }\end{array}\end{array}$ & 3 & 0,6 \\
$\begin{array}{l}\text { Perforation par roto- } \\
\text { percussion de la maçor } \\
\text { nerie (passage dur) }\end{array}$ & 2,5 & 0,1 à 0,9 \\
$\begin{array}{l}\text { Curage des fondations } \\
\text { existantes à l'aide } \\
\text { d'un marteau pneumati- } \\
\text { que léger }\end{array}$ & 1 & 2,4 \\
\hline
\end{tabular}

Tableau II

Ces résultats ont conduit à conseiller de supprimer la percussion et à interdire les perforations dans les zones situées au droit des toiles. Les fissures existantes, dont les mouvements étaient constamment surveillés en phase de travaux, n'ont présenté aucune évolution sensible qui puisse être mise sur le compte des vibrations. 


\section{III.3. Chevaux de MARLY}

Les "Chevaux de MARLY" comprennent quatre groupes sculptés, disposés sur le pourtour de la place de la Concorde, deux se trouvant à l'entrée des Champs Elysées, et les deux autres à l'entrée du Jardin des Tuileries. Les sculptures sont soumises aux agents atmosphériques, à la pollution de l'air, aux vibrations créées par la circulation urbaine et présentent un certain nambre de fissures. Le ministère de la Culture et de la Communication a souhaité contrôler les niveaux vibratoires dans des conditions de trafic usuelles et pour la sollicitation exceptionnelle que représente le défilé militaire du 14 Juillet.

Les points de mesures ont été implantés au sol, sur le haut du piedestal et sous le poitrail du cheval. Les vibrations les plus fortes ont été enregistrées sur la sculpture même, selon la direction horizontale perpendiculaire aux Champs Elysées, lors du passage des chars lourds et ont atteint un maximum de $6 \mathrm{~mm} / \mathrm{s}$. Les autres engins militaires ont produit des vibrations dont la résultante était inférieure ou égale à $3,8 \mathrm{~mm} / \mathrm{s}$.

Dans les conditions de trafic normal, les vibrations créées par les véhicules sont fonction de leur poids (voiture ou bus) et du cycle déterminé pour les feux tricolores règlant la circulation sur les Champs Elysées; les résultats sont les suivants (Tableau III):

\begin{tabular}{|c|c|}
\hline Source & $\begin{array}{l}\text { Vitesse résultante } \\
\text { sur cheval ( } \mathrm{mm} / \mathrm{s})\end{array}$ \\
\hline $\begin{array}{l}\text { Feu vert - passage } \\
\text { voitures }\end{array}$ & 0,3 \\
\hline Feu vert - passage bus & 0,7 \\
\hline $\begin{array}{l}\text { Freinage voitures au } \\
\text { feu orange }\end{array}$ & 0,5 \\
\hline
\end{tabular}

Tableau II I

Il est apparu que le maximum de vibrations se produit dans tous les cas sur le cheval qui amplifie essentiellement les composantes horizontales des vibrations arrivant du sol au travers du piedestal. Le rapport d'amplification global est en moyenne de 4,9 , soit 1,8 entre le sol et le piedestal et 2,7 entre le piedestal et la structure.

Compte tenu du cas particulier que représentent ces sculptures, le seuil admissible a été estimé à $3 \mathrm{~mm} / \mathrm{s}$. Dans ces conditions, le passage des chars lourds ou engins similaires présente un risque non négligeable. Quant à la circulation routière, son effet peut être considéré comme faible mais elle ne peut jouer à terme qu'un rôle défavorable sur la fissuration existante.

\section{III.4. Exemples divers}

Nous donnons ici quelques résultats provenant de cas classiques et pouvant servir d'ordre de grandeur pour le praticien :

\begin{tabular}{|c|c|c|}
\hline Source & $\begin{array}{c}\text { Distance } \\
(\mathrm{m})\end{array}$ & $\begin{array}{r}\text { Vitesse } \\
(\mathrm{mm} / \mathrm{s})\end{array}$ \\
\hline $\begin{array}{l}\text { Vibrofonçage d'un pro- } \\
\text { filé pour compactage } \\
\text { d'un remblai } \\
\text { Mesure sur plancher } \\
\text { d'un pavillon }\end{array}$ & 50 & 0,8 \\
\hline $\begin{array}{l}\text { Compactage dynamique } \\
\text { (type MENARD) } \\
\text { Masse de } 10 \mathrm{~T} \text { tombant } \\
\text { de } 10 \mathrm{~m} \text { de haut } \\
\text { Mesure dans un immeu- } \\
\text { ble d'habitation }\end{array}$ & 400 & $\begin{array}{l}0,05 \\
\text { à } \\
0,8\end{array}$ \\
\hline $\begin{array}{l}\text { Vibrofonçage de pal- } \\
\text { planches. } \\
\text { Mesure dans salle } \\
\text { d'ordinateur } \\
\text { Tour Manhattan (PARIS } \\
\text { La DEFENSE) }\end{array}$ & 70 & 0,4 \\
\hline
\end{tabular}

Tableau IV

Dans tous les cas cités, les vibrations créées étaient sans danger pour les ouvrages mais ont fait naitre l'inquiétude ou même la peur chez les occupants et les travaux ont do être suspendus de façon momentanée ou définitive.

\section{IV - SEUILS ADMISSIBLES ET NORMALISATION}

Une revue des normes et recommandations actuelles a été récemment donnée par CHAPEAU $(1980)$.

Les niveaux admissibles pour les individus sont maintenant bien connus :

- norme française : AFNOR E.90-400 (1971)

- norme internationale : ISO 2631 (1974) (amendement Janvier 1977).

Par contre, pour les batiments, les choses sont moins nettes; les cas possibles étant très variés, la normalisation est délicate. Les références actuellement les plus utilisées sont le projet de norme allemande DIN 4150 et les recommandations de 1'ATTES.

Nous donnerons ici des indications sur deux cas particuliers :

- ordinateur : pour un IBM 360 les seuils admissibles fournis par le fabricant sont 
donnés en fonction de l'accélération et ont les valeurs suivantes:

- vibrations entretenues : $0,1 \mathrm{~g}$

- vibrations interinittentes : $0,25 \mathrm{~g}$.

- tunnels (DOWDING et al 1978) : les tunnels sont beaucoup plus résistants aux vibrations que les constructions de surface. Des tirs d'essai ont été effectués aux ETATS UNIS au dessus de tunnels forés dans du grès, non revêtus, et de diamètres égaux à $2 \mathrm{~m}, 5 \mathrm{~m}$ et $10 \mathrm{~m}$. Des chutes de pierres occasionnelles se produisirent en général pour des vitesses moyennes de $120 \mathrm{~cm} / \mathrm{s}$. Dans le cas d'une galerie de $1,8 \mathrm{~m} \times 2,40 \mathrm{~m}$ perforée dans du terrain très schisteux, dont le soutènement était assuré par boulonnage et béton projeté, les premières fissures sont apparues pour des vitesses d'environ $91 \mathrm{~cm} / \mathrm{s}$.

Il ne s'agit là que de valeurs indicatives. De manière générale, on doit tenir compte dans l'évaluation du risque en tunnel d'un grand nombre de paramètres dont notamment, la nature du terrain encaissant et la qualité du contact terrain - revêtement.

\section{V - PREVISION DES NIVEAUX VIBRATOIRES}

Les facteurs à connaitre dans la prévision du niveau de vibrations sont liés aussi bien à la nature de la source qu'aux caractéristiques des milieux transmetteur et récepteur. C'est pourquoi il n'est pas envisageable de donner de règles générales valables partout. En pratique, on est conduit à utiliser à la fois les résultats fournis par la littérature, par des essais, et son jugement fondé sur l'expérience et l'estimation des risques encourus.

Dans le cas de tirs d'explosifs, il existe un certain nombre de règles faisant intervenir la charge instantanée et la distance:

- Chapeau (1980) donne une loi reliant Ia vitesse V, la charge Q et la distance D de la forme:

$$
\text { (1) } \quad v=k\left(\frac{D}{\sqrt{Q}}\right)^{m}
$$

où $m$ et $k$ sont des paramètres à établir expérimentalement. Une étude statistique générale conduit à l'estimation suivante :

- tir d'abattage :

$$
\text { (2) } V_{p} \max .<1,2(D / \sqrt{Q})^{-1,8}
$$

- tirs bloqués (bouchons, essais en forage)

$$
\text { (3) } \quad V_{p} \max .<2,4(D / \sqrt{Q})-1,8
$$

- Pour un site sensible, où une campagne de mesures complète a pu être menée, SIMECSoL a utilisé une loi de la forme :
(4)

pour le site considéré :

$$
\text { (5) } \begin{array}{rr}
V_{p}=0,758 Q^{0,612} & e^{-0,0070} \\
(\mathrm{~mm} / \mathrm{s}) & (\mathrm{Kg})
\end{array}
$$

- Dans le cas de tirs mettant en cause la stabilité d'un versant, STOTT (1978) propose une loi faisant intervenir l'accélération A, d'expression :

$$
A=k\left(\frac{D}{\sqrt[3]{Q}}\right)^{m}
$$

En pratique un seuil est choisi et les paramètres entrant dans la définition de lois sont déterminés d'après des tirs d'essais ; ensuite, est établi un abaque, fixant la charge admissible en fonction de la distance.

\section{VI - CONCLUSIONS}

A travers les quelques exemples cités, il apparait que les problèmes posés par les vibrations transmises par le sol, sont d'une grande variété tant dans leur nature que par leurs effets. C'est pourquoi, il est bien difficile de donner des règles de conduite ou des modèles de calcul. Chaque cas devra être étudié comme un cas particulier en se méfiant surtout de témoignages humains.

En effet, le corps est beaucoup plus sensible aux vibrations que les batiments; de plus, celles-ci peuvent avoir des effets sans danger mais spectaculaires (oscillations d'objets instables, déplacement de bibelots, etc...) produits à distance et parfois sans bruit. L'habitant voit naitre en lui un sentiment d'inquiétude souvent mal contrôlée et c'est à ce moment là qu'il se met à découvrir des fissures "nouvelles" qui ne font qu'aviver ses craintes.

Cette attitude fréquente peut déboucher sur l'arrêt complet des travaux, sans qu'il n'y ait danger véritable. Dans le cas des tirs d'explosifs, c'est bien souvent l'effet de surprise, le souffle et la détonation qui faussent les témoignages, même de bonne foi.

Une bonne façon d'agir est de se méfier de ses impressions personnelles et de faire les contrôles nécessaires, en vérifiant que les points et les directions de mesure sont adaptés au problème posé. Les résultats, connus en général immédiatement, permettent d'apprécier de façon objective le niveau de danger, et d'en déduire les dispositions à prendre. En matière de prévision, l'expérience acquise et une campagned'essais restent encore de nos jours, les seuls moyens dont on dispose. 
CHAPEAU C (1980) : "Le creusement des tunnels à l'explosif en site urbain".

Tunnels et Ouvrages souterrains $n^{\circ} 41$ Sept.0ct. $80-(266-275)$.

DOWDING CH, ROZEN A (1978) : "Damage to rock tunnels from earthquake shaking".

J. of the Geotech. Engng. Div (ASCE) Féb. 1980 GT2 (175-191).

HAIMSON BC (1978). "Effect of cyclic loading on rocks".

Dynamical Geotechnical Testing - ASTM STP $6541978(228-245)$.

STOTT MF (1979) : "A determination of the safe proximity for quarry blasting adjacent to slopes".

NCB Sept. 1979 ( 31-32-45). 